\title{
T. LIVII
}

\section{AB URBE CONDITA}

\author{
LIB. III-VI \\ QVAE SVPERSVNT IN CODICE RESCRIPTO VERONENSI \\ I)ESCRIPSIT ET EDIDIT
}

TH. MOMMSEN.

EX COMMENTATIONIBVS REGIAE ACADEMIAE SCIENTIARVM BEROLINENSIS

A. MDCCCLXVIII.

BEROLINI.

FORMIS ACADEMICIS

MDCCCLXVIII.

IN COMMISSIS LIBRARIAE F. DUEMMLERI

(IIARRWITZ ET GOSSMASN). 
Commentatio lecta in academicorum conventu d. xvi. Ian. sDccclxvili. Paginae quae indicantur voluminis sunt a. MDCcCLXviII actorum classis philologicae et historicae Regiae Academiae scientiarnm. 
V codex bibliothecae capitularis Veronensis n. 40.

sic ubi adscripsi, in re praesenti adnotavi codicis Veronensis scripturam vere ita esse ut refertur.

? litteram, cui signum superpositum est, indicat dubiam esse.

$\dddot{A}$ et similia significant lineolam, qua repraesentantur in fine versus litterae $m$ vel $n$, iam non cerni quidem, sed posse adfuisse.

I litteram indicat excidisse. Plerique autem hiatus indicati sunt non virgulis his, sed insertis quae exciderunt litteris forma diversa.

$M$ codex Mediceus (Laur. plut. 63,19) saec. XI ad haec quae edimus denuo recognitus mea causa a R. Schoellio. Varia lectio datur integra, nisi quod non adnotavimus nec orthographica nec minoris momenti errores scripturae primae in ipso libro emendatos: denique interpolationes manus secundae consulto omisi omnes.

$P$ codex Parisinus (n. 5725) saec. X secundum collationem Alschefskianam.

$L$ codex Leidensis (publ. Lat. fol. n. 6 A, Drakenborchii primus) a me excussus. Varia lectio datur plena similiter ac Medicei.

$M^{a}$ cet. codicis Medicei cet. prima scriptura.

$M^{b}$ cet. codicis Medicei cet. scriptura ex emendatione nata.

$C$ codices $M P L$ consentientes. 
